\title{
st \\ Um panfleto de Berkeley contra as práticas matemáticas de Newton e de Leibniz
}

\author{
Alex Galazans
}

O interesse de Berkeley pela matemática se manifestou desde sua juventude. Prova disso são muitas das entradas em seus cadernos de anotações, escritos entre 1706 e 1708 . Publicados postumamente, esses cadernos tornaram-se conhecidos como Philosophical commentaries (Comentários filosóficos). Ali encontramos afirmações que apresentam uma postura crítica tanto com relação à consagrada matemática clássica (cujos paradigmas se encontram nos Elementos de Euclides), quanto com relação ao novo cálculo infinitesimal. Por exemplo, na anotação 333, Berkeley avalia negativamente duas das mais celebradas novidades matemáticas de sua época: o método das fluxões de Newton e o cálculo diferencial de Leibniz. Outros textos seus reforçam a presença de um profundo interesse pelos assuntos matemáticos em sua juventude. Exemplos disso são a Arithmetica absque algebra aut Euclide demonstrata (A aritmética demonstrada sem a álgebra nem Euclides) e a Miscellanea mathematica (Miscelânia matemática), publicados em 1707 com o objetivo de obtenção da posição de fellow no Trinity College de Dublin. No curto ensaio Of infinites (Dos infinitos), texto entregue à Dublin Philosophical Society em 1707, Berkeley (cf. 2005) ataca novamente os novos métodos do cálculo infinitesimal. No entanto, interessa aqui apresentar outro texto sobre o mesmo assunto: The analyst (O analista), o qual é considerado um texto da maioridade filosófica de Berkeley. A publicação de sua primeira edição aconteceu durante o ano de 1734 e em duas cidades diferentes: inicialmente em Londres e, posteriormente, em Dublin. Somente um ano após sua morte, ocorrida em 1753, é que se concretiza a segunda edição.

Dada a localização temporal do aparecimento de 0 analista e os temas ali abordados, pode-se afirmar que Berkeley foi um dos principais personagens de duas grandes polêmicas de cunho matemático. Uma que independe de 0 analista e outra que depende totalmente dele. A primeira polêmica é independente, porque surgiu antes de 1734 . Trata-se do debate acerca da prioridade da invenção do "cálculo", que envolveu dois ilustres personagens do período: Newton e Leibniz. A outra polêmica depende intrinsecamente de $O$ analista, pois a pauta principal da discussão diz respeito aos assuntos 
que Berkeley ali tratou. Assim, cabe descrever qual é o objetivo dele ao publicar seu panfleto e qual é a relação entre as duas polêmicas.

Ao final de $O$ analista, Berkeley acrescenta uma longa sequência de questões acerca dos temas ali desenvolvidos. Em especial a Questão 66 descreve algo que pode indicar o motivo que o teria levado a elaborar tal texto: "questão 66. A analítica moderna não forneceria um forte argumentum ad hominem contra os atuais infiéis que cultivam a matemática?" Dois elementos chamam a atenção aqui. Um deles é que Berkeley se refere à analítica moderna. Trata-se daquela nova técnica matemática que procura solucionar problemas geométricos através da manipulação de equações. Descartes, com a obra A geometria (1637), é um dos mais expressivos representantes desse novo modo de fazer matemática. Os dois métodos, o das fluxões e o calculus differentialis, teriam sido elaborados a partir dessa nova perspectiva matemática. Por outro lado, ainda na mesma questão, Berkeley classifica os matemáticos como infiéis. Esse é o outro elemento que merece esclarecimento. De fato, o termo aponta para um problema teológico, sugerindo que um dos objetivos centrais de $O$ analista não seria propriamente matemático. Há questões que envolvem a religião e que forçaram Berkeley a escrever sobre assuntos matemáticos. Essa dupla temática já está explícita no título expandido do texto: "um discurso dirigido a um matemático infiel. Onde se examina se o objeto, os princípios e as inferências da análise moderna são mais distintamente concebidos ou mais obviamente deduzidos que os mistérios religiosos e as questões de fé”. Está evidente que Berkeley se propõe a apresentar ao infiel matemático uma comparação entre as pretensões cognitivas e argumentativas da religião e da matemática. Os elementos de comparação são a clareza de concepção (distinctly conceived) e a obviedade da dedução (evidently deduced) daquilo de que cada um desses âmbitos trata.

Mas o que teria levado Berkeley a querer realizar essa comparação? Uma das explicações mais conhecidas é aquela que Joseph Stock teria ajudado a propagar com a publicação de uma biografia de Berkeley em 1776, segundo a qual o matemático infiel ao qual Berkeley estaria fazendo referência é Edmund Halley, astrônomo que ficou conhecido por causa da descrição da órbita do famoso cometa que leva o seu nome. Samuel Garth, um amigo em comum com Berkeley, teria sido convencido por Halley a não receber os últimos serviços espirituais no leito de morte. Isso porque, para Halley, "as doutrinas do cristianismo são incompreensíveis e a religião em si uma fraude" (Berkeley, 1992, p. 131). Ao saber disso, Berkeley teria escrito 0 analista endereçado a Halley com o intuito de mostrar quão incompreensível seria a matemática em que os analistas modernos tanto confiavam. Muitos comentadores de Berkeley não confirmam essa explicação de Stock (cf. Jesseph, 1993, p. 179; Robles, 2006, p. 19). Sua principal dificuldade é que Berkeley estava na Itália quando Garth morreu em janeiro de 1719; e quem supostamente teria descrito o episódio da morte a Berkeley seria Joseph 
Um panfleto de Berkeley contra as práticas matemáticas de Newton e de Leibniz

Addison, que morreu em junho desse mesmo ano, de modo que Berkeley só poderia ter sido avisado por meio de cartas. Porém, não há qualquer indício da existência delas.

Mesmo diante da dificuldade de identificar quem seria o infiel matemático, há ainda outro aspecto que pode dar uma indicação da motivação de Berkeley para escrever $O$ analista, relacionando questões matemáticas e teológicas. No frontispício, Berkeley não acrescenta seu nome para indicar sua autoria; ele o faz de maneira indireta, apontado a autoria de outro texto seu: "Pelo autor de The minute philosopher". Trata-se de seu texto Alciphron, or The minute philosopher (Alcifron ou 0 diminuto filósofo), ${ }^{\mathbf{1}}$ publicado em 1732. Ali Berkeley ataca os livres-pensadores, que se tornaram notórios por rejeitarem a religião, acusando-a de conter elementos irracionais. Desse modo, não é por acaso que, em 0 analista, Berkeley faça referência ao Alciphron, pois os dois textos possuem, de modo geral, uma agenda comum: produzir um argumento contra aqueles que contribuem para o descrédito da religião. Em especial, em 0 analista, Berkeley utiliza a matemática, pois agora o alvo são matemáticos (ditos livrespensadores) que degradariam a religião ao compará-la com a comemorada evidência matemática. Mas de qual maneira a matemática é utilizada? Berkeley vê na própria matemática aceita por eles um meio para contra-atacar os "pretensos" raciocínios

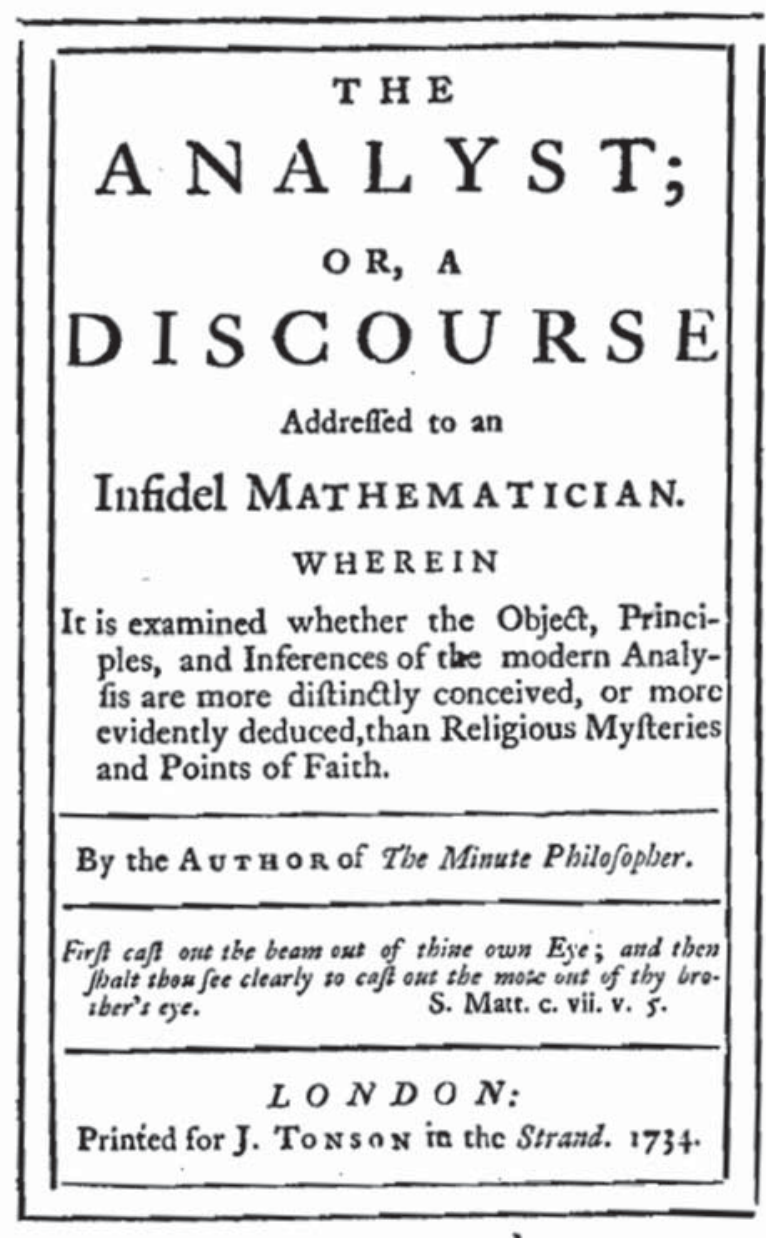

Frontispício da primeira edição de $O$ analista

$1 \mathrm{O}$ adjetivo minute presente no subtítulo de Alciphron é ambíguo e admite, ao menos, dois sentidos distintos. Ele pode ser sinônimo tanto de little ou small (pequeno, minúsculo) quanto de narrow (inflexível) ou careful (rigoroso, minucioso). Para Robles, tomado no segundo sentido, o minute de Berkeley pode aludir às pretensões de rigor e precisão expressas pelos livres-pensadores; enquanto que, no primeiro sentido, ele remeteria a impressão que Berkeley teria desses filósofos por se dedicarem a assuntos tão minúsculos, ao invés de utilizarem a razão para assuntos mais elevados (cf. 2006, p. 20). Robles opta por traduzir o subtítulo do Alciphron como el filósofo minucioso. $\mathrm{Na}$ falta de uma tradução do texto integral para a língua portuguesa, optou-se aqui por traduzir minute como minúsculo, que é o sentido mais frequentemente utilizado em comentários publicados em português. 
desfavoráveis à religião. Em outras palavras, para Berkeley, os livres-pensadores assumem a postura de "supremos senhores da razão" (cf. §1). Aqui está implícito que eles pretensamente querem argumentar que a disciplina merecedora de crédito, do ponto de vista racional, seria a matemática e não a religião, pois na matemática não se aceita nada que não seja racional, diferentemente dos assuntos teológicos que estão repletos de mistérios. Se existe um argumento nesse caso, para Berkeley, é algo que não se sustenta, porque é falso assumir a matemática como uma ciência livre de mistérios. Em $O$ analista, Berkeley quer mostrar que existem sim mistérios aceitos em seus métodos matemáticos, isto é, existem mistérios tanto no método das fluxões quanto no calculus differentialis. É por esse motivo que Berkeley se questiona: mas com que arremedo de razão poderia um homem pretender dizer que os mistérios não devem ser objetos de fé e, ao mesmo tempo, ele próprio admitir que tais mistérios obscuros sejam objetos de ciência? (cf. §7). Os próprios livres-pensadores, entretanto, não percebem que os seus métodos matemáticos estão repleto de problemas da mesma natureza que eles dizem existir na religião. Retoricamente, Berkeley sugere então, na questão 66, que a hipocrisia dos livres-pensadores daria ocasião a um sonoro argumento ad hominem. ${ }^{2}$

Por outro lado, para que sua própria argumentação também não fortalecesse um insidioso argumento ad hominem, Berkeley de fato teve que a sustentar em uma sólida perspectiva doutrinária que se mostra quando, na maior parte do texto, sua atenção volta-se para a avaliação das práticas matemáticas de Newton e Leibniz, focalizando os objetos e os princípios demonstrativos em cada caso. Isso mostra que, apesar de não ser considerado um matemático, Berkeley possuía um profundo conhecimento da matemática e que, principalmente, não estava alheio àquelas então recentes novidades produzidas nesse âmbito. Pode-se afirmar que a crítica matemática de Berkeley estrutura-se em dois domínios. Um que enfatiza a inteligibilidade dos objetos tratados em tais matemáticas e o outro que avalia a consistência lógico-demonstrativa de tais métodos.

Quanto aos objetos (\$3-8), Berkeley reprova a inteligibilidade do que Newton denominou momentos e do que Leibniz denominou diferenças. Ambos os objetos são recusados por Berkeley porque em suas definições estaria subentendido o apelo a entidades inacessíveis à imaginação - "faculdade essa derivada dos sentidos”. Para Ber-

\footnotetext{
2 Berkeley, obviamente, não está se referindo ao argumento ad hominem na forma mais conhecida, que consiste em se recusar a admitir um determinado argumento em virtude de determinadas particularidades (psicológicas, ideológicas, morais etc.) da pessoa que o apresenta. A modalidade a que ele se refere é, provavelmente, aquela conhecida como tu quoque, que consiste em concluir que a pretensão de uma determinada pessoa (no caso em questão, uma coletividade, os livres-pensadores) está equivocada porque é inconsistente com algo que ela tenha dito antes ou com suas próprias ações pregressas.
} 
keley, quer queiram quer não, Newton e Leibniz estariam negociando com quantidades infinitamente pequenas. Tais quantidades não suportam o critério berkeleyano de inteligibilidade. Nesse âmbito da crítica, é evidente que o critério utilizado faz parte da sua filosofia do esse est percipi (ser é ser percebido). Em O analista, a exemplo do Tratado sobre os princípios do conhecimento humano (1710), os legítimos objetos dos conhecimentos são ideias percebidas. O empirismo de Berkeley coloca os sentidos como a fonte inicial nesse processo de percepção. A imaginação, para atuar, dependeria necessariamente para atuar do que os sentidos fornecem, sendo ela incapaz de gerar por si mesma ideias abstratas a partir do que é oriundo dos sentidos. Os momentos e as diferenças são ininteligíveis, porque não são percebidos por nenhuma das faculdades mentais, sejam os sentidos seja a imaginação.

A situação torna-se ainda mais problemática na medida em que Newton e Leibniz fornecem uma representação simbólica para os momentos e as diferenças. Para Berkeley, a manipulação de símbolos converte-se em mais um empecilho para a compreensão de tais objetos matemáticos. É por isso que ele se pergunta na questão 26 de 0 analista: "a analogia e o emprego de signos têm sido suficientemente considerados pelos matemáticos? Até que ponto a restrita natureza específica das coisas corresponderia aos signos?". De fato, manipular os símbolos $\dot{x}, \ddot{x}, \dot{x}, \ddot{x}$ etc. (como no caso de Newton) e $d x, d d x, d d d x, d d d d x$ etc. (como no caso de Leibniz) não é, para Berkeley, suficiente para conferir inteligibilidade àquilo que se pretende representar com eles.

A segunda parte da crítica trata dos princípios demonstrativos (§9-20). Berkeley para isso apresenta duas demonstrações de Newton: aquela que se encontra no segundo lema do livro 2 dos Principia (Princípios matemáticos da filosofia natural) de 1687; e a outra, no manuscrito De quadratura curvarum de 1704. É na avaliação do segundo exemplo de demonstração, o do De quadratura, que Berkeley localiza um duplo tratamento dado ao conceito de momento. Ocorre que, no início das demonstrações, os momentos são apresentados como algo que é acrescentado e que, portanto, possui alguma magnitude. Porém, ao longo do que é demonstrado, os momentos passam a contar como algo destituído de qualquer magnitude, ou seja, passam a ser tratados como nada. Berkeley afirma que há uma mudança injustificada de hipótese no percurso da demonstração. Ao final de 0 analista, na questão 28, Berkeley cogita a possibilidade de os geômetras modernos cometerem a fallacia suppositionis (falácia da mudança de hipótese). Com efeito, para ele, é justamente no que Newton incorre com o seu duplo tratamento dos momentos.

Por outro lado, quanto ao calculus differentialis, Berkeley não se detém em qualquer uma de suas demonstrações como o fez no caso do método das fluxões, porque ele os compreende como semelhantes, principalmente quando se leva em consideração o objetivo de tais métodos: 
Pode não ser completamente incorreto observar que o método para encontrar a fluxão de um retângulo de duas quantidades fluentes, tal como se apresenta no De quadratura curvarum (Tratado das quadraturas), difere do acima mencionado retirado do segundo livro dos Principia, e que é, na prática, idêntico àquele usado no calculus differentialis $(\S 17)$.

Para Berkeley, é por isso que Leibniz incorre no mesmo problema demonstrativo de Newton:

No calculus differentialis, Leibniz e seus seguidores não têm qualquer escrúpulo para, em primeiro lugar, supor e, em seguida, rejeitar quantidades infinitamente pequenas, com uma clareza de compreensão e uma exatidão de raciocínio que poderia ser discernida por qualquer homem pensante isento de preconceitos $\left(\S_{18}\right)$.

Leibniz estaria cometendo o mesmo tipo de falácia de Newton, porque, ao supor uma quantidade infinitamente pequena no cálculo, para Berkeley, ela estaria sendo considerada como possuindo alguma magnitude. Porém, ao rejeitá-la, ela passa a ser tratada como não possuindo tal magnitude. Portanto, eis novamente uma injustificada mudança de hipótese.

O critério demonstrativo que Berkeley tem em mente para avaliar os métodos matemáticos de Newton e Leibniz é o da geometria euclidiana e que estaria pautada nos princípios da boa demonstração. É por isso que ele afirma já no início de 0 analista que considera a geometria como uma "excelente lógica". Desse modo, a acusação de fallacia suppositionis ocorre a partir de critérios que os próprios matemáticos estariam aceitando, ou seja, nesse âmbito da crítica, Berkeley está acusando tais matemáticos de não respeitarem os critérios demonstrativos aceitos por eles.

Todavia, resta saber como e por que, mesmo cometendo tão flagrantes equívocos lógicos, tanto o método das fluxões quanto o calculus differentialis obtêm resultados verdadeiros. A tese de Berkeley é que ali ocorre uma compensação de erros (\$21-29).

No restante do texto ( $\$ 30-50)$, Berkeley constrói réplicas a possíveis interpretações que seriam dadas em favor de tais métodos matemáticos. É possível nesse percurso conectar a crítica de Berkeley, como um todo, a temas que são centrais em sua filosofia. Por exemplo, no parágrafo 31, Berkeley se recusa a admitir que exista velocidade independente da relação espaço e tempo. O que está em jogo é a impossibilidade de tratar a velocidade de maneira abstrata - isto é, destituída de suas determinações espaciais e temporais. Isso nada mais é do que um exemplo de seu antiabstracionismo, algo que aparece em outras obras suas, como na introdução do Tratado sobre os princípios do conhecimento humano. 
Contudo, dentre outras questões, uma ainda merece atenção. Trata-se do posicionamento de Berkeley quanto à polêmica entre Newton e Leibniz. O assunto principal dessa polêmica era a prioridade da invenção da solução de alguns problemas matemáticos tratados por eles. Por exemplo, eles elaboraram (e hoje se sabe que foi independentemente um do outro) novas soluções para determinar a tangente e a quadratura de curvas. Mas, principalmente, perceberam que tais problemas eram inversos um ao outro (o que atualmente, no cálculo diferencial e integral, é conhecido como teorema fundamental do cálculo). O desenvolvimento de tal polêmica ocorre durante o final do século xviı e primeira metade do século xviı. Temas, como o problema da fundamentação matemática, foram utilizados por newtonianos e leibnizianos para apontar defeitos ou virtudes dos métodos no desenvolvimento dessa polêmica. O próprio Newton chegou a afirmar durante a polêmica algo nesse sentido:

Este método [das fluxões] deriva imediatamente de sua própria natureza, não de indivisíveis, diferenças leibnizianas ou quantidades infinitamente pequenas. Pois não existem quantidades primeiras nascentes ou quantidades últimas evanescentes, existem somente razões primeiras de quantidades nascentes e razões últimas de quantidades últimas evanescentes (Whiteside, 1967-1980, v. 3, p 17-8).

O que está em questão é que, para Newton, o método das fluxões estaria mais bem fundamentado matematicamente, pois se apoiaria em um conceito de primeiras e últimas razões; o que se caracterizaria como uma tentativa de evitar o apelo a quantidades infinitamente pequenas. Newton se mostra convencido da impossibilidade ontológica de tais quantidades e, para ele, o método de Leibniz seria inferior quanto a sua fundamentação por utilizar tais quantidades. Por sua vez, Berkeley manifesta ter conhecimento da disputa ocorrida entre os dois matemáticos. Isso se verifica, por exemplo, no parágrafo 18, de 0 analista. No entanto, a essa altura, pode-se afirmar que Berkeley não faz a defesa nem de Newton nem de Leibniz, uma vez que ambos os métodos são reprovados quanto à inteligibilidade de seus objetos e quanto à consistência de suas demonstrações. Portanto, Berkeley se recusa a dar a vitória a qualquer um deles, em virtude dos problemas similares relativos aos fundamentos dos métodos.

Considera-se que a publicação de 0 analista, em 1734, dá início, principalmente na Grã-Bretanha, a um movimento de esclarecimento dos aspectos relacionados ao rigor matemático do cálculo infinitesimal (cf. Guicciardini, 1989, p. 38; Robles, 2006, p. 279). Nesse sentido, o que entrou em questão foi o estatuto ontológico dos objetos matemáticos e a "correção lógica" das demonstrações matemáticas de acordo com os cânones demonstrativos da geometria euclidiana (cf. Calazans, 2008, p. 391-418). Figuram dentro desse movimento as respostas de James Jurin e John Walton, que fo- 
ram os primeiros a responder às críticas de Berkeley. Jurin, com o pseudônimo de Philalethes cantanbrigiensis, ainda em 1734, escreve o Geometry no friend to infidelity; or a defence of Sir Isaac Newton and the British mathematicians (A geometria não é amiga da infidelidade; ou uma defesa de Sir Isaac Newton e dos matemáticos britânicos); e Walton, no ano seguinte, escreve Vindication of Sir Isaac Newton's Principles offluxions (Reivindicação dos princípios das fluxões de Sir Isaac Newton). Berkeley interveio nessa discussão somente mais duas vezes. Em $17^{3} 5$, ele escreve Defence of free-thinking in mathematics (Defesa do livre-pensamento em matemática) como resposta a Jurin, incluindo somente um apêndice como resposta a Walton. O outro texto de Berkeley foi Reasons for not replying to Mr. Walton's full answer (Razões para não responder à resposta completa do Sr. Walton), escrito no ano de 1735 em resposta à segunda reação de Walton desse mesmo ano, intitulada The catechism of the author of the "Minute Philosopher" fully answer'd (O catecismo do autor de "O diminuto filósofo" completamente respondido). No caso de Jurin, ele escreveu como resposta à réplica de Berkeley o texto The minute mathematician; or, the free-thinker no just-thinker (O diminuto matemático; ou o livre-pensador e não só-pensador). ${ }^{3}$ Supõe-se que Berkeley não teve acesso a esse segundo texto de Jurin, resumindo-se, dessa maneira, somente àquelas duas primeiras tréplicas a sua participação direta nessa polêmica. É difícil delimitar até onde se estende o debate desencadeado por Berkeley. Presume-se que, no final da primeira metade do século xviı, quase todos os textos publicados na Inglaterra a respeito da matemática fazem alguma menção ao texto de 0 analista (cf. Jesseph, 1993, p. 231-95).

Nomes como os de Benjamin Robins, John Colson, Roger Paman e Colin Maclaurin aparecem ainda como ativos participantes da polêmica. Um exemplo muito interessante é o caso de Maclaurin. Ele escreve, em 174,2, o texto Treatise offluxions (Tratado das fluxões). No prefácio da obra Maclaurin afirma que foi a leitura de O analista que o motivara a escrever seu texto. Há, no entanto, comentadores que alertam para os perigos de superestimar essa afirmação de Maclaurin, pois seu tratado não teria sido exclusivamente escrito para responder a Berkeley, mas, principalmente, para divulgar muitas das novidades matemáticas elaboradas pelo próprio Maclaurin (cf. Grabiner, 1997). Por outro lado, na introdução, Maclaurin faz uma comparação entre o método das fluxões e os métodos matemáticos que utilizam quantidades infinitamente pequenas. Segundo ele, o método matemático de Newton foi construído a fim de evitar as quantidades infinitamente pequenas, constituindo-se em um "caminho muito mais satisfatório à exatidão (strictness) da geometria" (Maclaurin, 1801 [1742], v. 1, p. 2). É evidente que Maclaurin está se referindo à polêmica entre Leibniz e Newton. No en- 
tanto, o objetivo agora é o de defender a superioridade do método newtoniano. Isso permite afirmar que Maclaurin não ignorou as duas polêmicas matemáticas do período. Sua resposta a Berkeley teria que dar conta, ao mesmo tempo, de problemas que envolviam a polêmica anterior e a polêmica posterior ao texto de 0 analista. Dentro dessa perspectiva, pode-se concluir que o trabalho de Berkeley, em seu panfleto, além de permitir uma melhor compreensão de alguns aspectos centrais de sua própria filosofia (que concernem não só a sua filosofia da matemática), deve ser encarado também como uma fonte primária de estudo da fortuna do confronto que opôs newtonianos e leibnizianos, representando um divisor de águas no pensamento matemático inglês do século xviıi.@

\section{Alex Calazans}

Doutorando do Programa de Pós-Graduação em Filosofia, Instituto de Filosofia e Ciências Humanas, Universidade Estadual de Campinas, Brasil. filoalexcalazans@gmail.com

\section{REFERÊNGIAS BIBLIOGRÁFIGAS}

Apolônio de Perga. Conics, Tradução W. H. Donahue. Santa Fe: Green Lion, 1998.

Berkeley, G. L'analyste. Tradução A. Leroy. Paris: PUF, 1936.

The analyst. In: Luge, A. A. \& Jessop, T. E. (Ed.). The works of George Berkeleybishop of Cloyne. Nendeln:

Nelson (Kraus Reprint), 1979. v. 4, p. 53-102.

The analyst. Introdução e notas Douglas Jesseph. In: Jesseph, D. (Ed.) De motu and The analyst: a modern edition, with introductions and commentary. Dordrecht e Boston: Kluwer Academic Publishers, 1992. (New Synthese Historical Library, n. 42).

. L’analyste. Tradução M. Blay. In: Brykman, G. (Ed.) Oeuvres. Paris: PUF, 1999. v. 2, p. 257-332. Dos infinitos. Tradução Jean Rodrigues Siqueira. Trans/Form/Ação, 28, 2, p. 47-51, 2005.

. El analista. Tradução e notas de J. A. Robles. In: Robles, J. A. Los escritos matemáticos de George Berkeley y la polémica sobre El Analista. México: Unam/Instituto de Investigaciones Filosóficas, 2006. p. $55^{-104}$.

. De motu. Sobre o movimento ou sobre o princípio, a natureza e a causa da comunicação dos movimentos. Tradução M. R. da Silva. Scientiae Studia, 4, 1, p. 115-37, 2006a.

Brykman, G. (Ed.). Oeuvres. Paris: PUF, 1999. 2 v.

Calazans, A. Considerações a respeito do problema do rigor matemático em "O analista" de Berkeley. Cadernos de História e Filosofia da Ciência, 18, 2, p. 391-4,18, 2008.

Conte, J. Obras filosóficas: George Berkeley. Tradução, Apresentação e notas J. Conte. São Paulo: Unesp, 2010.

Grabiner, J. Was Newton's Calculus a Dead End? The Continental Influence of Maclaurin's Treatise of Fluxions. American Mathematical Monthly, 104, 1997, pp. 393-4110. 
Guicciardini, N. The development of the Newtonian calculus in Britain: 1700-1800. Cambridge: Cambridge University Press, 1989.

Jesseph, D. M. Berkeley's Philosophy of Mathematics. Chicago/London: The University of Chicago Press, 1993.

. Berkeley's Philosophy of Mathematics. In: Winkler, K. (Ed.) The Cambridge Companion to Berkeley. Cambridge: Cambridge University Press. 2005, p. 266-310.

Luge, A. A. \& Jessop, T. E. (Ed.). The works of George Berkeley bishop of Cloyne. Nendeln: Nelson (Kraus Reprint), 1979.4 v.

Maclaurin, C. A treatise on fluxions. London: William Baynes/William Davis, 1801 [1742]. 2 v.

Robles, J. A. Los escritos matemáticos de George Berkeley y la polémica sobre El analista. México: Unam/ Instituto de Investigaciones Filosóficas, 2006.

Whiteside, D. T. (Ed.). The mathematical papers of Isaac Newton. Cambridge: Cambridge University Press, $1967-1980.8 \mathrm{v}$. 